\title{
Fusion Estimation of Point Sets from Multiple Stations of Spherical Coordinate Instruments Utilizing Uncertainty Estimation Based on Monte Carlo
}

\author{
Fumin Zhang, Xinghua Qu \\ State Key Laboratory of Precision Measuring Technology and Instruments, Tianjin University, Tianjin, 300072, China, \\ zhangfumin@tju.edu.cn
}

\begin{abstract}
Multiple instrument stations, based on spherical coordinate measurements, are often used in the measurement of large objects. A data fusion method is proposed to derive optimal estimations of the positions of the object features, measured by more than one device. First, each device has a dedicated coordinate system that is linked together through the measurement of common points. Second, the weighted mean coordinates are derived. The covariance matrix of the sensory, covering of the radial distance and the angles, is propagated to get a weight matrix. Third, a nonlinear function is minimized to determine the optimized coordinate of the points. Monte Carlo error propagation is utilized to estimate the uncertainty of the fusion points. Simulation of the fusion algorithms is performed using laser tracking and laser radar. The fusion algorithm experiments are performed using two laser tracking stations. Simulation and experiments prove that the fusion method improves the precision of the measurements of an object's location, due to incorporating the degree of uncertainty for each measurement point.
\end{abstract}

Keywords: Large volume metrology, multiple-station data fusion, uncertainty, Monte Carlo, laser tracker

\section{INTRODUCTION}

$\mathrm{L}$ ARGE VOLUME metrology is increasingly required in fields such as commercial airplane production and shipbuilding. Commonly used instruments include laser trackers, electronic theodolites, photogrammetry systems and laser radar. Industrial applications necessitate either a combination of various measurement devices or the relocation of a single instrument throughout the measurement process in order to acquire complete data [1]. The foremost reason for using a combination of measurement systems is to overcome physical constraints. Moreover, in a large field of targets rich with obstructions, line of sight is often necessary. Given a limited measurement range, it is not always possible to measure the entire object using a single measurement system. The diversity in individual measurement system properties offers further reason to use a combination of measurement systems. Systems have varying degrees of accuracy, working volume, measurement speed, setup time and cost. In order to achieve the highest degree of efficiency, these limitations and features must be considered when designing a measurement process.

The combination of full coordinate measurement systems may also be used to achieve greater accuracy than what could be achieved by the use of a single sensor alone. Users often combine measurement systems by linking individual measurement systems together based on common reference points. When given multiple sets of measurements for the same set of targets, a combined estimate of the target locations should be obtained. The topic of multi-sensor data fusion has received a lot of attention from the scientific community, for both industrial and non-industrial applications. In applying those techniques, one would expect to achieve benefits such as extending the system's spatial/temporal coverage, improved detection performance and enhanced resolution (spatial/temporal) [2-3].
Llinas described an application where a moving aircraft is observed by both a pulsed radar system and an infrared imaging sensor [4]. El-Hakim combines models created from multiple images and range sensors [5]. He also uses known shapes, CAD drawings, existing maps, survey data, and GPS data. It has been shown that the use of intensity data produced by a range camera can improve the accuracy of vision-based 3-D measurements. Wendt et al. presented an approach for data fusion and simultaneous adjustment of inhomogeneous data intended to increase the accuracy and reliability of surface reconstruction. Their study is based on 3-D data obtained from fringe projections and photogrammetry-based systems [6]. Johnson et al. described a technique for adaptive resolution surface generation from multiple distributed sensors. They demonstrated the technique using 3-D data generated by a scanning lidar and a structure from a motion system [7]. J. Angelo Beraldin et al. discussed the integration of laser scanning and closerange photogrammetry with a multi-sensor and information fusion point of view. They presented the key features of different laser scanner technologies and photogrammetrybased systems that should be considered in order to realize the benefits expected from a multi-sensor platform [8-9]. Joseph M. Calkins used five total stations in conjunction with three laser trackers for a measurement job of a submarine [10]. Considering the low distance uncertainty in laser trackers, multi-path laser tracker measurement systems have been studied by several researchers such as Zhang GX, Zhuang Hanqi, Zhang Defen [11-12]. Forbes AB combined measurements from the laser tracker and the theodolite [13]. C. Read Predmore studied a Bundle adjustment technology of multi-position measurements using the Mahalanobis distance [14]. A Weckenmann reviewed multi-sensor data fusion in dimensional metrology [15].

This paper is organized as follows: Section 2 presents the weight mean and nonlinear fusion method from multi- 
station measurements, Section 3 proposes the uncertainty of each measurement point for fusion, using the Monte Carlo method, Section 4 gives an introduction to the simulation test evaluation procedure, using laser trackers and laser radar, Section 5 details the analysis of the experiment results using two laser tracker stations, and finally conclusions are given in Section 6.

\section{FUSION METHODS}

It is assumed that point $\boldsymbol{D}=\left[x^{*}, y^{*}, z^{*}\right]^{T} \in \mathfrak{R}^{3}$ represents 3$\mathrm{D}$ true coordinates of the location of a large sized object. However, the true values are unknown and our aim is to derive estimators $\hat{\boldsymbol{D}}=\left[\hat{x}^{*}, \hat{y}^{*}, \hat{z}^{*}\right]^{\mathrm{T}}$ of $\boldsymbol{D}$, from available multiple devices or stations of one device $\mathrm{D}_{i} i=1, \ldots, n$. It is assumed that $\boldsymbol{\theta}_{i} \in \mathfrak{R}^{p}$ denotes the data collected by the $i$-th station, and $p$ is the number of sensors in one station. $p=3$ is considered in this paper. The mathematical model $f$ of each measurement station is used to convert sensor data $\boldsymbol{\theta}$ to coordinate $\boldsymbol{D}$. In general, $f$ is nonlinear. In particular, a spherical coordinate measurement instrument is considered in this paper. Let $\boldsymbol{\theta}^{*}=\left(l^{*}, \alpha^{*}, \beta^{*}\right)^{T}$ be the point $\boldsymbol{D}$ expressed spherically, where $l$ is the radial distance from the coordinate system origin, $\alpha$ is the horizontal angle, and $\beta$ is the vertical angle. The output of device $D_{i}$ is a random variable, $\boldsymbol{\theta}_{i}=\left(l_{i}, \alpha_{i}, \beta_{i}\right)^{T}$. Then a simple model of the random and systematic effects associated with the measurements $\boldsymbol{\theta}$ is given by:

$$
\begin{aligned}
& l_{i}=l^{*}+d_{0}+\varepsilon_{l i} \\
& \alpha_{i}=\alpha^{*}+\varepsilon_{\alpha i} \\
& \beta_{i}=\beta^{*}+\varepsilon_{\beta i}
\end{aligned}
$$

where, $\varepsilon_{l i} \sim N\left(0, \sigma_{l i}^{2}\right)$,

$$
\begin{aligned}
& \varepsilon_{\alpha i} \sim N\left(0, \sigma_{\alpha i}^{2}\right), \\
& \varepsilon_{\beta i} \sim N\left(0, \sigma_{\beta i}^{2}\right), i=1, \ldots, n .
\end{aligned}
$$

Independent, random effects $\varepsilon=\left(\varepsilon_{l}, \varepsilon_{\alpha}, \varepsilon_{\beta}\right)^{T}$ are varied from point to point, while systematic effect $d_{0}$ represents fixed offsets associated with the laser dead path of the laser interferometer. The systematic effect can be corrected with the procedures provided by the manufacturer.

The data in the spherical coordinates is converted to Cartesian 3-D coordinates with the $f$ as (2) to obtain from the i-th station:

$$
\boldsymbol{D}_{i}=f\left(\boldsymbol{\theta}^{*}+\varepsilon_{i}\right)=\left(\begin{array}{c}
l_{i} \cos \alpha_{i} \sin \beta_{i} \\
l_{i} \sin \alpha_{i} \sin \beta_{i} \\
l_{i} \cos \beta_{i}
\end{array}\right)
$$

The task is to derive good estimators $\hat{\boldsymbol{D}}$ from $\boldsymbol{\theta}_{i}=\left(l_{i}, \alpha_{i}, \beta_{i}\right)^{T}, i=1, \ldots, n$.

Firstly, all measuring data points from the multiple stations should be unified to the same reference coordinate system from their respective coordinate systems, using the rigidity coordinate transform. This problem is a classic task that could be called absolute orientation. The transformation between two Cartesian coordinate systems can be thought of as the result of a rigid-body motion. Therefore, the transformation parameters can be decomposed into a $3 \times 3$ rotation matrix $\boldsymbol{R}$ and a $3 \times 1$ translation vector $\boldsymbol{T}$. There are three degrees of freedom to translation vector $\boldsymbol{T}$. Rotation matrix $\boldsymbol{R}$ has another three degrees of freedom (direction of the axis about which the rotation takes place plus the angle of rotation about this axis). Singular Value Decomposition (SVD) presents a mature closed-form solution to the least-squares problem of absolute orientation [16]. The process sets a reference coordinate system with any single station, while transforming the others. Typically, this would be the system with the lowest uncertainties, the most recently calibrated, or the most centrally located system with respect to the object being measured.

The weighted average of the coordinates from $n$ stations is obtained using (3)

$$
\hat{\boldsymbol{D}}=\sum_{i=1}^{n} \boldsymbol{W}_{i} \boldsymbol{D}_{i}
$$

where $\boldsymbol{W}_{i} \in \mathfrak{R}^{3 \times 3}$ is the $i$-th station weighted matrix that can be determined by assigning less weight to coordinates with higher uncertainties. For example, because uncertainty of the measurements taken using a laser tracker is considerably better for range than it is for angle, the distance-derived measurements are assigned greater weight than the anglederived measurements [17]. The uncertainty associated with a measured coordinate $\boldsymbol{D}_{i}$ can be represented in terms of covariance matrix $\boldsymbol{V}_{\mathrm{i}}$, that is a $3 \times 3$ symmetric, positive (semi)-definite matrix. The diagonal elements of $\boldsymbol{V}_{\mathrm{i}}$ are the variances associated with the coordinates, and the offdiagonal elements are the covariances. The standard uncertainty associated with a single coordinate is the square root of the corresponding diagonal element. Often, $\boldsymbol{V}_{\mathrm{i}}$ can be derived from sensor covariances $\boldsymbol{V}_{\varepsilon i}$, that is a diagonal variance matrix of the noise $\varepsilon_{i}$. If the square deviation is independent, the covariance matrix $\boldsymbol{V}_{\varepsilon i}$ associated with sensors in each station can be written as:

$$
\boldsymbol{V}_{\varepsilon}=\left(\begin{array}{ccc}
\sigma_{l}^{2} & & \\
& \sigma_{\alpha}^{2} & \\
& & \sigma_{\beta}^{2}
\end{array}\right)
$$

The uncertainties associated with these effects are propagated through to those associated with the Cartesian coordinates as in (5).

$$
V_{i}=J_{i} V_{\delta \theta i} J_{i}^{T}
$$

Where $\boldsymbol{J}$ is the Jacobian matrix of $f$ with respect to $\boldsymbol{\theta}$. Taylor's expansion is calculated for (1) as:

$$
\boldsymbol{D}_{i}=f\left(\boldsymbol{\theta}^{*}\right)+\boldsymbol{J}_{i} \boldsymbol{\varepsilon}_{i}=\left(\begin{array}{c}
l^{*} \cos \alpha^{*} \sin \beta^{*} \\
l^{*} \sin \alpha^{*} \sin \beta^{*} \\
l^{*} \cos \beta^{*}
\end{array}\right)+\boldsymbol{J}_{i} \boldsymbol{\varepsilon}_{i}
$$

The Jacobian matrix can be solved by: 


$$
\boldsymbol{J}_{i}=\frac{\partial f}{\partial \boldsymbol{\theta}}=\left(\begin{array}{lll}
\frac{\partial x}{\partial l} & \frac{\partial x}{\partial \alpha} & \frac{\partial x}{\partial \beta} \\
\frac{\partial y}{\partial l} & \frac{\partial y}{\partial \alpha} & \frac{\partial y}{\partial \beta} \\
\frac{\partial z}{\partial l} & \frac{\partial z}{\partial \alpha} & \frac{\partial z}{\partial \beta}
\end{array}\right)=\left(\begin{array}{ccc}
\cos \alpha_{i} \sin \beta_{i} & -l_{i} \sin \alpha_{i} \sin \beta_{i} & l_{i} \cos \alpha_{i} \cos \beta_{i} \\
\sin \alpha_{i} \sin \beta_{i} & l_{i} \cos \alpha_{i} \sin \beta_{i} & l_{i} \sin \alpha_{i} \cos \beta_{i} \\
\cos \beta_{i} & 0 & -l_{i} \sin \beta_{i}
\end{array}\right)
$$

Therefore the weight matrix can be determined as (8):

$$
\boldsymbol{W}_{\boldsymbol{i}}=\left[\sum_{i=1}^{n} \boldsymbol{V}_{i}^{-1}\right]^{-1} \boldsymbol{V}_{i}^{-1}
$$

Using derived weighted matrix and (3), the initial estimation of the fusion coordinate $\hat{\boldsymbol{D}}$ is derived.

Although uncertainty is considered in the weighted matrix, this approach also disregards another uncertainty. For example, the SVD method explained above ignores that the uncertainties associated with the measured coordinates for each data point are anisotropic in the presence of errors $\varepsilon$. In addition, most uncertainties of the systematic effect are not considered. Even if some systematic effect can be corrected, remaining error also has uncertainty. Therefore, the fused estimate $\hat{\boldsymbol{D}}$ and the parameters of the transformation matrices are optimized by solving nonlinear function (9)

$$
\min _{\hat{\boldsymbol{D}}, \boldsymbol{R}, \boldsymbol{T}} \sum_{i=1}^{n}\left(\boldsymbol{D}_{\boldsymbol{i}}-\hat{\boldsymbol{D}}\right)^{T} \boldsymbol{W}_{\boldsymbol{i}}\left(\boldsymbol{D}_{\boldsymbol{i}}-\hat{\boldsymbol{D}}\right)
$$

As explained above, using $\boldsymbol{W}_{\mathrm{i}}$ also represents individual weight relative to all effects. Therefore residual error between multiple $\boldsymbol{D}_{i}$ can be minimized by this function. This problem can be posed as a nonlinear, least-squares problem that can be solved by the Levenberg-Marquardt minimization algorithm.

\section{ESTIMATION OF MEASURING UNCERTAINTIES BASED ON MONTE CARLO SIMULATIONS}

Uncertainty evaluation is a critical part of multi-sensor data fusion techniques. These include not only the uncertainty of the individual instruments' measurements, but the uncertainty of their registration and fusion to the other instruments. The covariance matrix of optimized coordinates can be computed as:

$$
\boldsymbol{V}=\left(\boldsymbol{J}^{T} \boldsymbol{J}\right)^{-1}
$$

However, it is difficult to obtain the Jacobian matrix in a nonlinear function optimization process [18]. Therefore, the GUM appendix gives a framework for the expression of the measurement uncertainty based on the Monte Carlo (MC) method [19-20]. The fundamentals of this technique are that the uncertainty of a particular coordinate measurement is simulated using knowledge of the position of the measurement instrument and the uncertainty of sensors in the instrument. The MC method repeats simulated measurements, each consisting of the nominal measurement value with random noise of the sensor added to it. Therefore, the MC method can propagate the probability density function (PDF) of each input quantity, from each coordinate acquisition station, directly to the total measurement set through the measurement model [21-23].

Each series of measurements for all the points from a single measurement station represents one point group around the nominal point, which is illustrated as scatter. The uncertainty scatter is a graphical representation of the uncertainty of each measurement, and additionally allows the uncertainty of the measurement to be estimated by directly measuring the dispersion of these points. A graphical representation of the uncertainty is helpful for properly representing the uncertainty interactions of nonlinear fusion from multiple stations [24].

The steps of the uncertainty estimation of the fusion value from $\mathrm{MC}$ can be summarized as follows:

(1) Select a number $N$ of trials.

(2) Generate a random draw $\boldsymbol{\theta}_{1 i}$ for $i$-th station input quantity $\boldsymbol{\theta}^{*}$ from its assigned PDF.

(3) Propagate the random value $\boldsymbol{\theta}_{1 i}$ to obtain the output quantity value $\boldsymbol{D}_{1 i}=f\left(\boldsymbol{\theta}_{1 i}\right)$. For each station except the first, all associated measurements are transformed to fusion $\hat{\boldsymbol{D}}_{1}$.

(4) By repeating the process $N$ times, the repeated transformation and fusion generate a composite scatter $\hat{\boldsymbol{D}}_{j} j=1, \ldots, N$.

(5) Take the mean of these values of $\hat{\boldsymbol{D}}_{j}$ as the measurement result $\hat{\boldsymbol{D}}$.

(6) Take the standard deviation of the $\hat{\boldsymbol{D}}_{j}$ as the standard uncertainty $\boldsymbol{u}_{\boldsymbol{D}}$ of the fusion measurements $\hat{\boldsymbol{D}}$ from multiple stations.

The large number $N$ and heavy density of the scatter diagram will take much operational time, while a few points could not reflect the uncertainty accurately [25]. To determine the adequate number of trials $N$ to produce stable results, different numbers of trials can be selected. The relation between the uncertainty analysis and scatter diagram density by simulation is shown in Fig.1. Therefore, we should choose an appropriate value, but not many times over. In the framework of this study, approximately 500 trials were found to be sufficiently stable. 


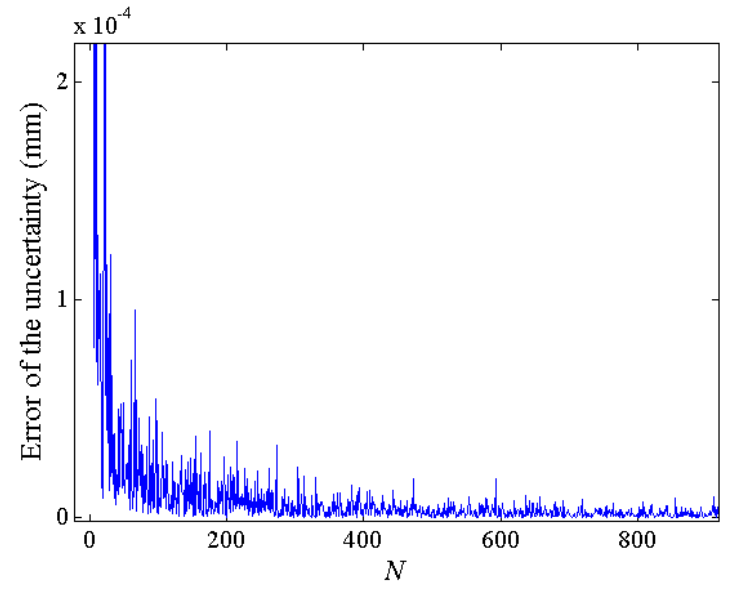

Fig.1. Simulation analysis of scatter diagram density.

\section{Simulation}

In order to test the fusion method, simulated data from laser radar and laser trackers was generated. The actual position of the measurement instrumentation in the simulated measurement configuration is placed at $(0,0,0)^{\mathrm{T}}$. Typically, laser radar and laser trackers make measurements of the target coordinates in spherical coordinates, with uncertainties of the range and two angles that vary as a function of the range. The nominal position of the measured point coordinate is set as

$$
\left(l^{*}, \alpha^{*}, \beta^{*}\right)^{T}=\left(1000,-45^{\circ}, 45^{\circ}\right)^{T} .
$$

The actual point was randomly deviated from the ideal point with a random error that has a standard deviation of $\sigma$.

The laser tracker provides an accurate estimate of the range with relative uncertainties of $\sigma_{l}=10 \mu \mathrm{m}+l \cdot 0.8 \mu \mathrm{m} / \mathrm{m}$, but with poor angular direction estimates and with uncertainty of $\sigma_{\alpha}=\sigma_{\beta}=1^{\prime \prime}$. Instead, the laser radar determines the angular direction with a higher than standard angular uncertainty of $\sigma_{\alpha}=\sigma_{\beta}=0.5^{\prime \prime}$, when compared to the laser tracker. The standard deviation for the uncertainty in the range was $0.3 \mathrm{~mm}$ plus $1 \mathrm{ppm}$. If these two observations are correctly associated, then the combination of the two sensors' data provides an improved determination of location in comparison to results that could be obtained by either of the two independent instruments.

The uncertainty scatter diagrams for the same measuring point using the laser radar measurements alone, laser tracker measurements alone and the combination of both, are shown in Fig.2, 3, and 4, respectively.

Uncertainty scatters indicate directly the strength of the fusion analysis. The standard uncertainty of point from the laser tracker station is $u_{D 1}=0.02 \mathrm{~mm}$. Standard uncertainty of point from the laser radar station is $u_{D 2}=0.10 \mathrm{~mm}$. Standard uncertainty of point from fusion method is $u_{D 3}=0.01 \mathrm{~mm}$.

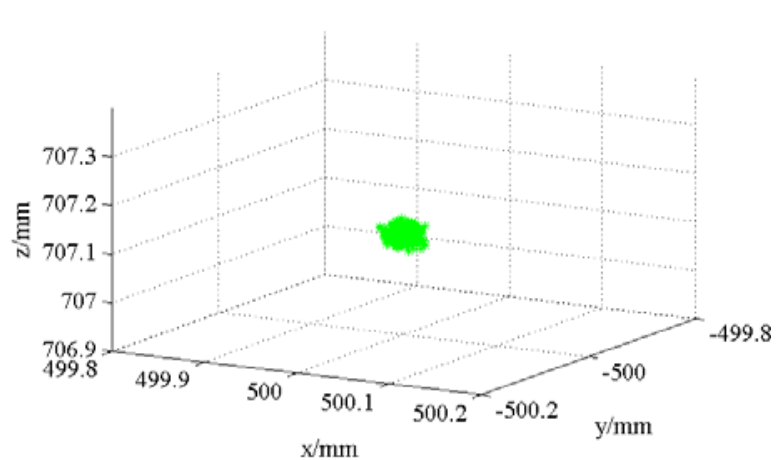

Fig.2. Uncertainty scatter diagram of laser tracker.

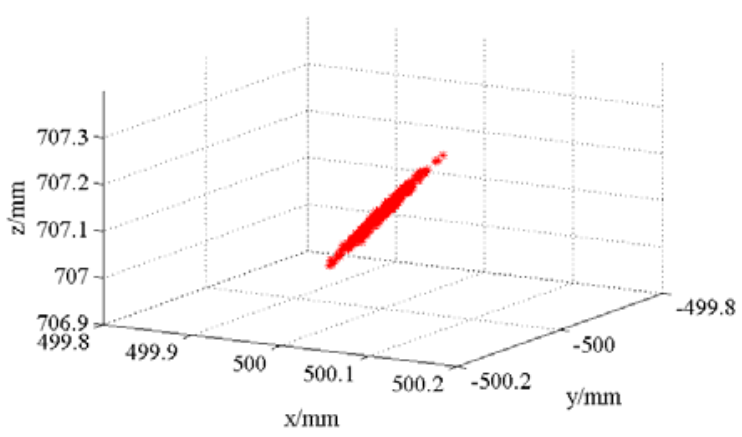

Fig.3. Uncertainty scatter diagram of laser radar.

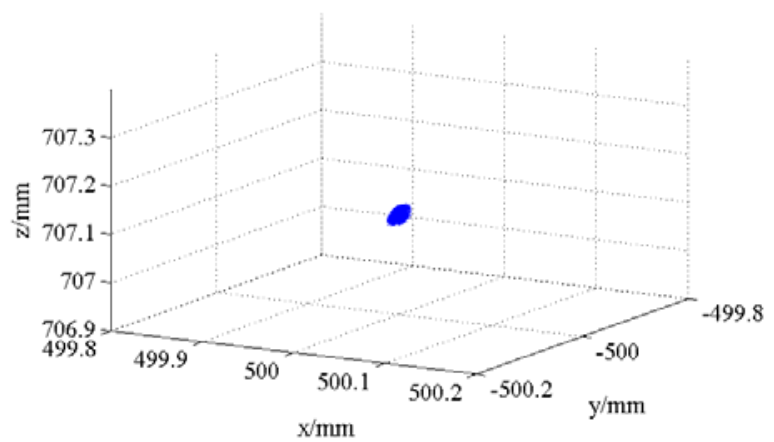

Fig.4. Uncertainty scatter diagram of fusion coordinate.

\section{PRACTICAL EXPERIMENTS}

An experiment was conducted with the FARO Xi laser tracker, which was placed in two positions to measure 5 points that covered a $5 m \times 5 m \times 2 m$ metrology volume.

The data was analyzed using techniques based on the covariance matrix to get result $S_{1}$, and using the MC method to get $S_{2}$, as shown in Table1.

Results show that the difference between $S_{1}$ and $S_{2}$ is small enough, therefore the MC method is an accurate covariance matrix. In addition, uncertainty of fusion coordinates is lower than with any single station. 
Table 1. Results of two station fusion using covariance matrix and $\mathrm{MC}(\mu \mathrm{m})$

\begin{tabular}{llrrr}
\hline & Number & \multicolumn{1}{c}{$s_{1}$} & \multicolumn{1}{c}{$s_{2}$} & \multicolumn{1}{c}{$s_{1}-s_{2}$} \\
\hline Station 1 & 1 & 4.04 & 3.99 & 0.05 \\
& 2 & 1.75 & 1.75 & 0.00 \\
& 3 & 10.59 & 10.86 & -0.27 \\
& 4 & 7.02 & 6.83 & 0.18 \\
& 5 & 17.94 & 17.94 & 0.00 \\
Station 2 & 1 & 1.33 & 1.30 & 0.03 \\
& 2 & 1.79 & 1.83 & -0.05 \\
& 3 & 3.14 & 3.24 & -0.10 \\
& 4 & 61.77 & 61.68 & 0.09 \\
Fusion & 5 & 17.71 & 17.94 & -0.23 \\
& 1 & 1.22 & 1.17 & 0.05 \\
& 2 & 1.16 & 1.20 & -0.04 \\
& 3 & 2.95 & 3.07 & -0.11 \\
& 4 & 6.92 & 6.79 & 0.14 \\
& 5 & 12.55 & 12.68 & -0.13 \\
\hline
\end{tabular}

\section{CONCLUSiOnS}

This paper describes the methodology for deriving the optimal estimation of the point sets from several spherical coordinate instrument locations. All data in each station was transformed into a common coordinate system based on the SVD method. The measuring model was established. The individual variance matrix associated with a spherical coordinate measurement system is propagated to the covariance matrix associated with measured point coordinates, to get its weighted matrix for averaging the data that was initially measured. Furthermore, a nonlinear optimized mean is used for deriving optimized coordinates to minimize residual error. By statistically processing each observation, the MC method can be used to estimate the overall uncertainty, to avoid the complex Jacobian Matrix. We illustrate the method to simulate measurements using laser trackers and laser radar. Results show that standard uncertainty of point from $0.02 \mathrm{~mm}$ and $0.10 \mathrm{~mm}$ with the laser tracker station and the laser radar station, respectively, was reduced to $0.01 \mathrm{~mm}$ with the fusion method. Two laser tracker stations were used in this experiment. We can conclude that the fusion method provides an improved degree of precision over simply averaging measured values. The MC method is easily applicable for evaluating the uncertainty of weighted combined coordinates.

Future research will concentrate on extending the fusion method to handle other non-spherical coordinate measurement instruments, such as vision metrology.

\section{ACKNOWLEDGMENT}

This study was supported by the National Natural Science Foundation of China (Grant No. 51105274), and Tianjin Research Program of Application Foundation and Advanced Technology (No. 11JCYBJC01000).

\section{REFERENCES}

[1] Estler, W.T., Edmundson, K.L., Peggs, G.N., Parker, D.H. (2002). Large-scale metrology - an update. CIRP Annals-Manufacturing Technology, 51 (2), 587-608.

[2] Luo, R.C., Yih, C.-C., Su, K.L. (2002). Multisensor fusion and integration: Approaches, applications, and future research directions. IEEE Sensors Journal, 2 (2), 107-119.

[3] More, K., Ingman, D. (2008). Quality approach for multi-parametric data fusion. NDT\&E International, 41 (3), 155-162.

[4] Hall, D.L., Llinas, J. (1997). An Introduction to multisensor data fusion. Proceedings of the IEEE, 85 (1), 6-23.

[5] El-Hakim, S.F. (2001). Three-dimensional modeling of complex environments. In Videometrics and Optical Methods for Three Dimensional Shape Measurement, 20-26 January 2001. SPIE Vol. 4309, 162-173.

[6] Wendt, A., Rosing, C., Weisensee, M. (2002). Comparison of different sensor types and validation of an approach for multi sensor fusion. In Close-Range Imaging, Long-Range Vision, 2-6 September 2002, 105-109.

[7] Johnson, A.E., Manduchi, R. (2002). Probabilistic 3D fusion for adaptive resolution surface generation. In IEEE Proceedings of the International Symposium on $3 D$ Data Processing Visualization and Transmission, 19-21 June, 2002, 578-587.

[8] Luhmann, T. (2010). Close range photogrammetry for industrial applications. ISPRS Journal of Photogrammetry and Remote Sensing, 65 (6), 558-569.

[9] Beraldin, J.A. (2004). Integration of laser scanning and close-range photogrammetry - the last decade and beyond. In Proceedings of the XXth ISPRS Congress, 12-23 July, 2004, 972-983.

[10] Joseph, M.C. (2002). Quantifying Coordinate Uncertainty Fields in Coupled Spatial Measurement Systems. Doctor of Philosophy, Virginia Polytechnic Institute, Virginia, United States of America.

[11] Zhuang, H.Q., Motaghedi, S.H., Roth, Z.S., Bai, Y. (2003). Calibration of multi-beam laser tracking systems. Robotics and Computer Integrated Manufacturing, 19 (4), 301-314.

[12] Zhang, D.F., Rolt, S., Maropoulos, P.G. (2005). Modelling and optimization of novel laser multilateration schemes for high-precision applications. Measurement Science and Technology, 16 (12), 2541-2547.

[13] Forbes, A.B., Hughes, B., Sun, W.J. (2009). Comparison of measurements in co-ordinate metrology. Measurement, 42 (10), 1473-1477.

[14] Predmore, C.R. (2010). Bundle adjustment of multiposition measurements using the Mahalanobis distance. Precision Engineering, 34 (1), 113-123.

[15] Weckenmann, A., Jiang, X., Sommer, K.D., Neuschaefer-Rube, U., Seewig, J., Shaw, L., Estler, W.T., (2009). Multisensor data fusion in dimensional metrology. CIRP Annals - Manufacturing Technology, 58 (2), 701-721. 
[16] Brujic, D., Ristic, M. (1997). Monte Carlo simulation and analysis of free-form surface registration. Proceedings of the Institution of Mechanical Engineers, Part B: Journal of Engineering Manufacture, 211 (8), 605-617.

[17] Mitchell, J.P., Spence, A.D., Hoang, M., Free, A. (2003). Sensor fusion of laser trackers for use in largescale precision metrology. In Proceedings of SPIE Photonics East, Vol. 5326, 57-65.

[18] Mana, G., Pennecchi, F. (2007). Uncertainty propagation in non-linear measurement equations. Metrologia, 44 (3), 246-250.

[19] Joint Committee for Guides in Metrology (2008). JCGM 101 - Evaluation of Measurement Data Supplement 1 to the "Guide to the Expression of Uncertainty in Measurement" - Propagation of Distributions Using a Monte Carlo Method. First Edition.

[20] International Organisation for Standardisation. (1995). Guide to the Expression of Uncertainty in Measurement. Second Edition.
[21] Balsamo, A., Ciommo, M.D., Mugno, R., Rebaglia, B.I., Ricci, E., Grella, R. (1999). Evaluation of CMM uncertainty through Monte Carlo simulations. Annals of the CIRP, 48 (1), 425-428.

[22] Kuhinek, D., Zorić, I., Hrženjak, P. (2011). Measurement uncertainty in testing of uniaxial compressive strength and deformability of rock samples. Measurement Science Review, 11 (4), 112-117.

[23] Lira, I. (2011). Monte Carlo evaluation of the uncertainty associated with the construction and use of a fitted curve. Measurement, 44 (10), 2156-2164.

[24] Kosarevsky, S.V., Latypov, V.N. (2012). Practical procedure for position tolerance uncertainty determination via Monte-Carlo error propagation. Measurement Science Review, 12 (1), 1-7.

[25] Dhanish, P.B., Mathew, J. (2006). Effect of CMM point coordinate uncertainty on uncertainties in determination of circular features. Measurement, 39 (6), 522-531. 